\title{
Beamforming in Intelligent Randomly Distributed Sensor Networks using Electrically-Small Dual-Sector Antennas for Planetary Exploration
}

\author{
Nicholas C. Soldner, Chunwei Jethro Lam, Andrew C. Singer and Jennifer T. Bernhard \\ Department of Electrical and Computer Engineering \\ University of Illinois at Urbana-Champaign \\ Urbana, IL 61801 \\ nsoldner@uiuc.edu, clam@uiuc.edu, acsinger@uiuc.edu, jbernhar@uiuc.edu
}

\begin{abstract}
Dependence on a small set of surface rovers for surface and atmospheric exploration exposes missions to higher than acceptable probabilities of failure. To mitigate this situation, we have developed enabling technology for small, low power, low cost sensors that can be scattered from multiple orbiters or secondary craft and form networks. Since antenna size is expected to determine wireless sensor feasibility, an electrically small dual-sector antenna is developed which gives $100 \mathrm{MHz}$ of 2:1 VSWR bandwidth from $2.61 \mathrm{GHz}-2.71 \mathrm{GHz}$ while occupying only $4 \mathrm{~cm}^{3}$. Sixteen of these antennas are placed randomly on a flat ground plane to determine the ability of the antenna network to transmit and receive data cooperatively from multiple angles. Performance is gauged by taking bit error rate measurements for various numbers of elements and angles. The beamforming algorithm presented is capable of establishing reliable communications regardless of relative positions and orientations of individual sensors.
\end{abstract}

\section{Introduction}

Given the hazardous conditions often found in atmospheric and surface based exploration, it is unwise to rely on a single sensor or a sensor system with a single point of failure such as a base station. If that system should fail, then the mission will also fail. An immediate solution to this problem is to utilize a redundant system in which many small sensors are deployed, where, if some sensors should fail, the mission would not. This approach to atmospheric and surface sensing missions breaks away from the conventional method in which all efforts are put into making a single piece of hardware failsafe. Using multiple sensors mitigates mission risk by reducing the probability of failure due to hardware.

When atmospheric or surface sensing probes are de- ployed by a remote vehicle, be it an orbiting spacecraft or aircraft, the probes will always be distributed randomly. Once in a state of rest, the probes will communicate with each other, collect data, and work together to transmit results in the direction of an orbiting spacecraft or aircraft. In order for this system to be viable, the sensors themselves must be small in order to keep payload weight and occupied volume to a minimum. The primary contributor to individual probe size will be the antenna, therefore, use of an electrically small antenna is desired to keep the sensor as small as possible. Also necessary are beamforming algorithms which allows the random sensor array to collectively steer a beam towards an orbiter or a ground based station. These two enabling technologies, development of an electrically small antenna and beamforming algorithms, are the focus of the present related work.

Development of an electrically small antenna based on the stacked sector design [1] is presented, as well as its use in a randomly deployed sensor network. To approximate a randomly deployed sensor network a test bed is designed in which sixteen antennas are randomly oriented on a ground plane. The ability of the random antenna array to beamform a signal is determined as the ground plane angle is varied. Network performance is determined by measuring the bit error rate (BER) as the angle between the deployed sensors and the orbiter varies, signal-to-noise ratio varies and for different numbers of nodes (sensors).

\section{Electrcally small stacked sector antenna}

The antenna developed for each sensor is based on the electrically small inductively-loaded stacked sector antenna [1]. An example of the electrically small stacked sector antenna is shown in Figure 1. This antenna is very low profile and is well suited to applications where 


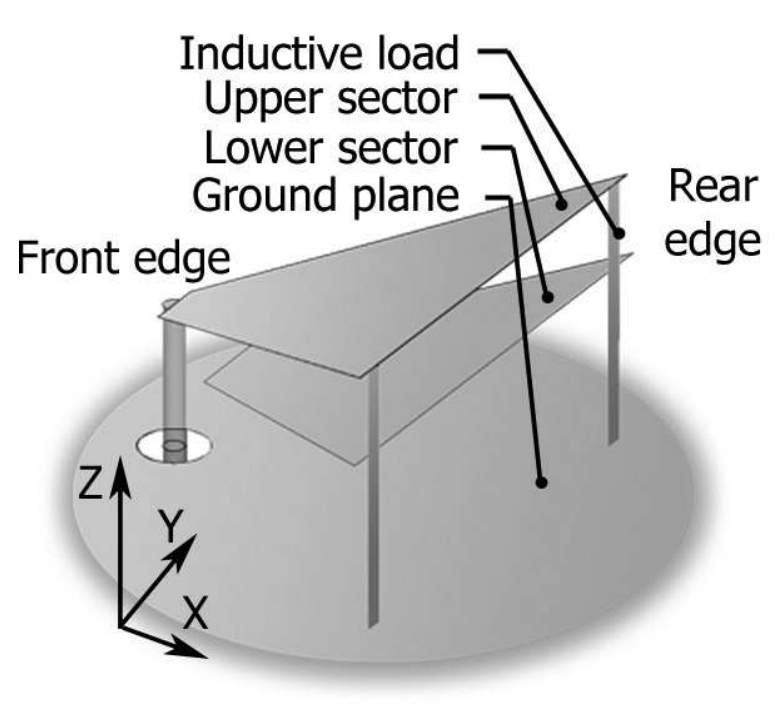

Fig. 1. Electrically small stacked sector antenna showing probe feed penetrating ground plane

space is very limited. Similar to a patch antenna in which the radiating elements are close and parallel to the ground plane, the stacked sector antenna differs by having elements, which are at a slight angle with the ground plane. The principal feature of the electrically small stacked sector antenna (ESSSA) is its use of multiple sectors to create multiple resonances. When the antenna is designed such that both resonances are closely spaced, impedance bandwidth is increased. Antenna size is further reduced through the use of inductive loads which are placed across the antenna aperture. By adding inductive loads the operating bandwidth is shifted downward in frequency without increasing physical size, effectively making the antenna look electrically larger. One inherent downfall to electrically small antennas is a very low real input impedance. For the stacked sector antenna an inductive feed was developed which alleviates the low input impedance problem and provides a $50 \mathrm{Ohm}$ match [2]. Using the inductive feed also removes the need for a matching transformer, further reducing space requirements for the antenna system.

Design goals for the antenna were an operating frequency of $1 \mathrm{GHz}-3 \mathrm{GHz}, 5$ - $20 \%$ 2:1 VSWR impedance bandwidth, occupy no more than $4 \mathrm{~cm}^{3}$, and exhibit isotropic radiation. It is important that the antenna have an isotropic radiation pattern so that it can communicate with other sensors and orbiters regardless of its orientation. Given the complexity of this design, closed form design equations, which give exact dimensions for the antenna given a desired operational frequency and bandwidth, are impractical. Development of the antenna was

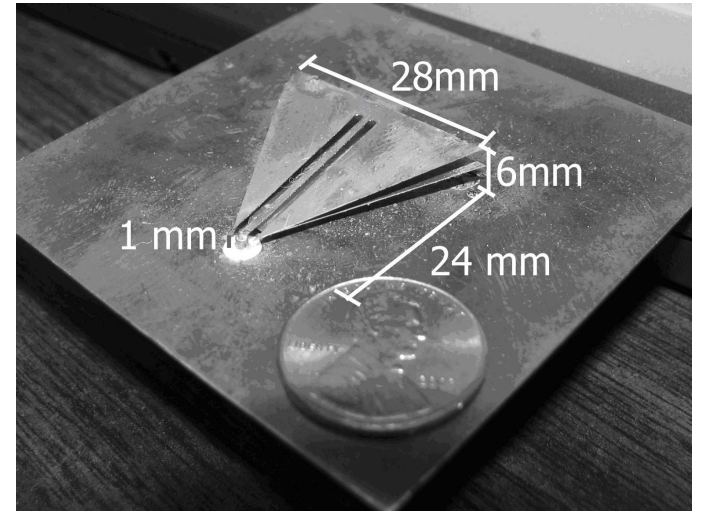

Fig. 2. Fabricated electrically small stacked sector antenna showing inductive feed integrated into upper sector

done primarily through parametric simulations using the High Frequency Structure Simulator (HFSS ${ }^{\circledR}$ ) [2, 3]. There many degrees of freedom for the stacked sector antenna: sector areas, inductive load widths, cant angle (the angle sectors make with the ground plane), sector flare angle, sector height above ground plane, and inductive feed length/width. Parametric simulations were developed in HFSS $^{\complement}$ ) which allowed the antenna response to be studied as one geometrical parameter was varied $[2,3]$. In a recursive process where the range of values in each parametric study was refined, an antenna which realized the design goals was created. The simulated design filled a $4 \mathrm{~cm}^{3}$ volume with the upper sector feed $1 \mathrm{~mm}$ from the ground plane and rear edge 6 $\mathrm{mm}$ high and the rear edge of the lower sector equidistant between the upper sector and ground plane. Simulations gave values for the inductive feed dimensions which were $0.9 \mathrm{~mm}$ wide with a $2.05 \mathrm{~mm}$ spacing on either side of the feed and an overall length of $20 \mathrm{~mm}$. The antenna was fabricated using a precision milling machine which uses geometry data directly exported from (HFSS $^{\complement}$ ). Both sectors and loads were fabricated from brass and the $65 \times 65 \mathrm{~mm}$ ground plane was constructed from copper (Figure 2).

The fabricated antenna efficiency was measured using a vector network analyzer in conjunction with the Wheeler cap method and was found to be $65 \%$ [4]. The fabricated antenna dimensions are shown in Figure 8. Figures 6 and 7 compare the HFSS simulated and fabricated designs and clearly there is good agreement between the results with measured gains only 1 $2 \mathrm{dBi}$ below simulated gains. The fabricated design has a VSWR $<2$ bandwidth of $100 \mathrm{MHz}(3.8 \%$ ) from 2.61 $\mathrm{GHz}-2.71 \mathrm{GHz}$ and in terms of wavelength the antenna is roughly $1 / 20 \lambda$ in height and $1 / 5 \lambda$ in length from feed 


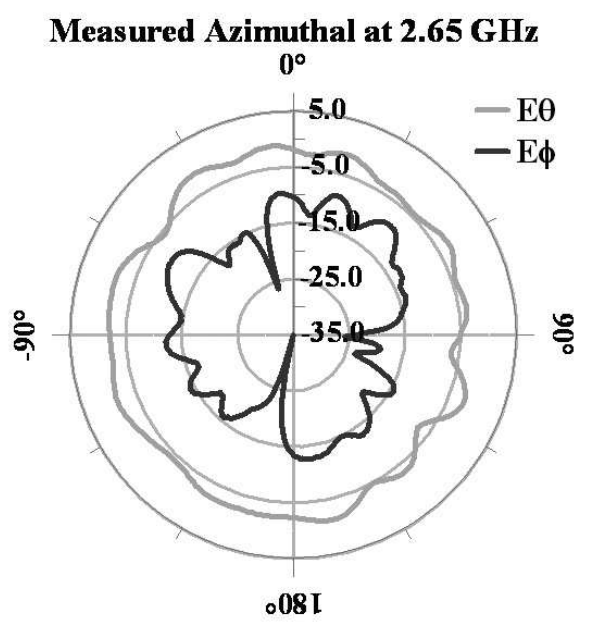

Fig. 3. Azimuthal radiation pattern

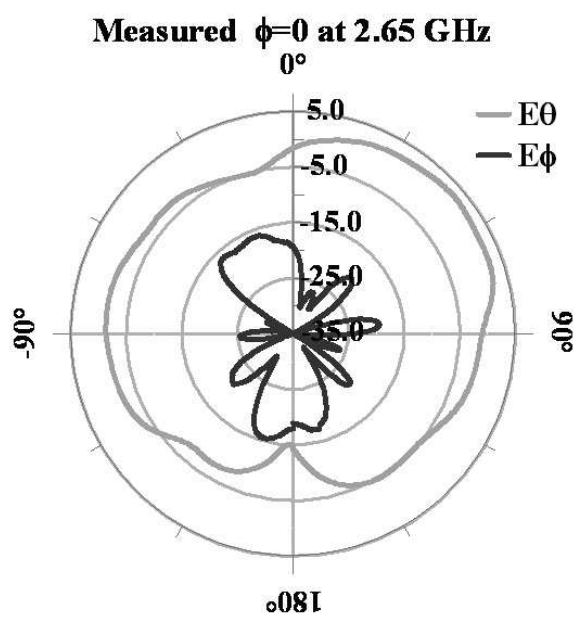

Fig. 4. $\phi=0$ radiation pattern

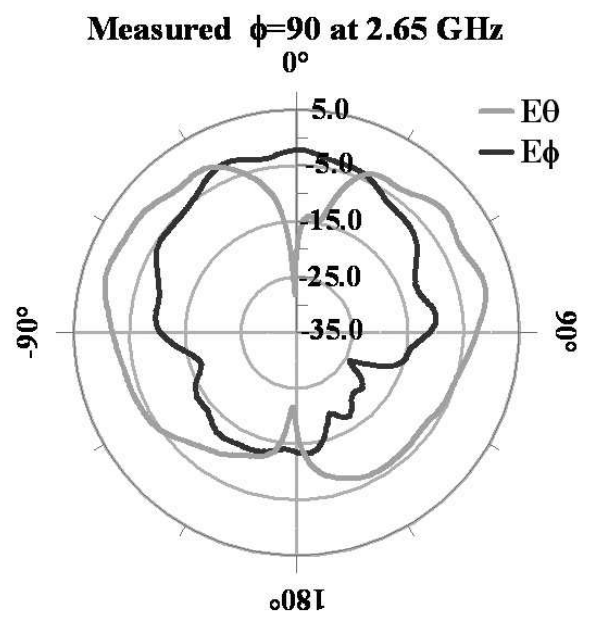

Fig. 5. $\phi=90$ radiation pattern

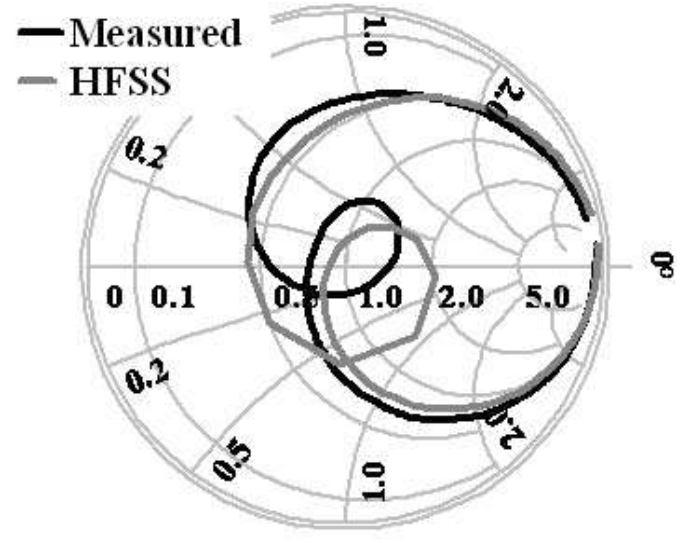

Fig. 6. Smith chart plot comparing fabricated antenna and simulated antenna

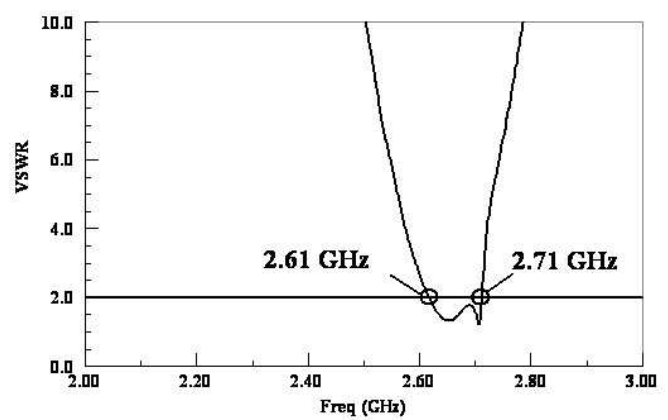

Fig. 7. Fabricated antenna VSWR plot showing 2.61 $\mathrm{GHz}-2.71 \mathrm{GHz}$ match bandwidth (bandwidth $=3.8 \%$ )

to rear edge. Antenna pattern plots are shown in Figures 3,4 , and 5 where it is clear the antenna has a radiation pattern similar to that of a dipole. All results were from the $65 \times 65 \mathrm{~mm}$ ground plane; previous simulations show that as the ground plane decreases in size VSWR 2:1 bandwidth decreases only slightly and the resonance associated with the lower sector and ground plane shifts. Since it is uncertain how large the antenna ground plane will be when finally installed in a sensor, simulations are carried out to determine the effects on antenna performance as ground plane size varies. Although ground plane size did effect the resonant frequencies of the antenna, the shift in resonant frequencies can be compensated by adjusting the lower sector cant angle.

\section{Beamforming Algorithms for Random Sensor Arrays}

The beamforming algorithm is responsible for establishing communications between the sensor network and the remote transmitter/receiver, e.g., an orbiter or an air- 


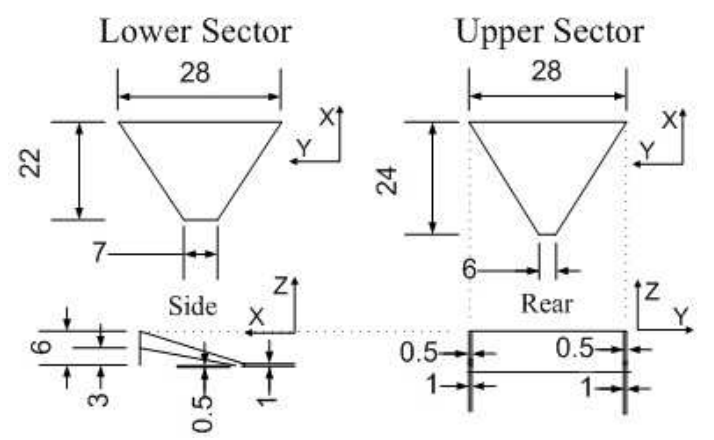

Fig. 8. Fabricated antenna dimensions (mm)

craft. The sensor nodes are interconnected wirelessly such that data is shared freely among the nodes. The relative positions and orientations of the sensors with respect to the orbiter are unknown and are assumed to be invariant in time.

The algorithm is composed of two stages. In the first stage, the orbiter transmits a beacon signal to the sensors, and the sensors estimate the spatial signature that characterizes the transmission direction. In the second stage, a receive beamformer is computed based on the spatial signature. The same strategy is then used to set up the transmit beamformer using reciprocity.

\subsection{Signal Model}

The orbiter transmits an RF signal

$$
\boldsymbol{\operatorname { R e }}\left\{s^{*}(t) e^{j 2 \pi f t}\right\}
$$

where $s(t)$ is the source, $f$ is the carrier frequency, $(\cdot)^{*}$ stands for complex conjugate, and $\operatorname{Re}\{\cdot\}$ stands for the real part. The RF signal received at the $n$th sensor is given by

$$
\boldsymbol{\operatorname { R e }}\left\{s^{*}\left(t+\Delta t_{n}\right) g_{n} e^{j\left(2 \pi f t+\theta_{n}\right)}\right\},
$$

where $g_{n}$ is the gain, $\Delta t_{n}$ is the time delay, and $\theta_{n} \triangleq$ $2 \pi f \Delta t_{n}$ is the phase delay. The parameters $g_{n}, \Delta t_{n}$ and $\theta_{n}$ collectively characterize the effects of path loss, polarization, the radiation patterns of the antennas, and the relative positions and orientations of the sensors with respect to the orbiter. Assuming that the source waveform is slow-varying within the time duration $\Delta t_{n}$, then $s(t)=s\left(t+\Delta t_{n}\right)$ is a valid approximation. In this case, the complex baseband signal is given by

$$
s^{*}(t) g_{n} e^{j \theta_{n}} .
$$

There are $N$ sensors in the network. Under additive white Gaussian noise, the received complex baseband signals can be written as an $N \times 1$ column vector

$$
\mathbf{x}(t)=s^{*}(t) \mathbf{a}+\mathbf{n}(t),
$$

where a stands for the spatial signature, or steering vector, whose column elements are $\left\{g_{1} e^{j \theta_{1}}, \ldots, g_{N} e^{j \theta_{N}}\right\}$, and $\mathbf{n}(t)$ is an $N \times 1$ noise vector independent of the source. The complex baseband signals are sampled at $t=k T$ with $T$ being the sampling period. This yields

$$
\mathbf{x}(k)=s^{*}(k) \mathbf{a}+\mathbf{n}(k) .
$$

Note that the sampling times of all sensors do not need to match exactly because the timing error is naturally absorbed in the definition of $\Delta t_{n}$. However, the sampling frequency, $1 / T$, must be identical for all sensors.

\subsection{Calibration}

In the first stage, the spatial signature a is measured by the sensors via observing a beacon signal that is known to all sensors a priori. Let $\left\{s_{b}(i)\right\}_{i=1}^{K}$ be the $K$ beacon data samples. A state space model can be written as

$$
\begin{aligned}
& \mathbf{a}(k)=\mathbf{a}(k-1) \\
& \mathbf{x}(k)=s_{b}^{*}(k) \mathbf{a}(k)+\mathbf{n}(k) .
\end{aligned}
$$

A Kalman filter is constructed to estimate the state via the recursions [5]

$$
\begin{aligned}
& \hat{\mathbf{a}}(k)=\hat{\mathbf{a}}(k-1)+G(k)\left(\mathbf{x}(k)-s_{b}^{*}(k) \hat{\mathbf{a}}(k-1)\right) \\
& G(k)=P(k-1) s_{b}^{*}(k)\left(\left|s_{b}(k)\right|^{2} P(k-1)+R_{n}\right)^{-1} \\
& P(k)=\left[\mathbf{I}-P(k-1)\left(P(k-1)+R_{n}\right)^{-1}\right] P(k-1)
\end{aligned}
$$

where $\mathbf{I}$ is the identity matrix and $R_{n}$ is the noise covariance. The latter can be measured from the sensors when the beacon signal is absent. The recursions stop when all the $K$ beacon samples are used.

\subsection{Beamforming}

In the second stage, an $N \times 1$ receive beamformer $\mathbf{w}$ is selected such that the beamforming output,

$$
y(k)=\mathbf{x}^{H}(k) \mathbf{w},
$$

recovers the source signal $s(k)$. Note that $(\cdot)^{H}$ stands for conjugate transpose. A common choice of $\mathbf{w}$ is the minimum variance distortionless response (MVDR) beamformer that has the form [6]

$$
\mathbf{w}=\mu \hat{R}^{-1} \mathbf{a} .
$$




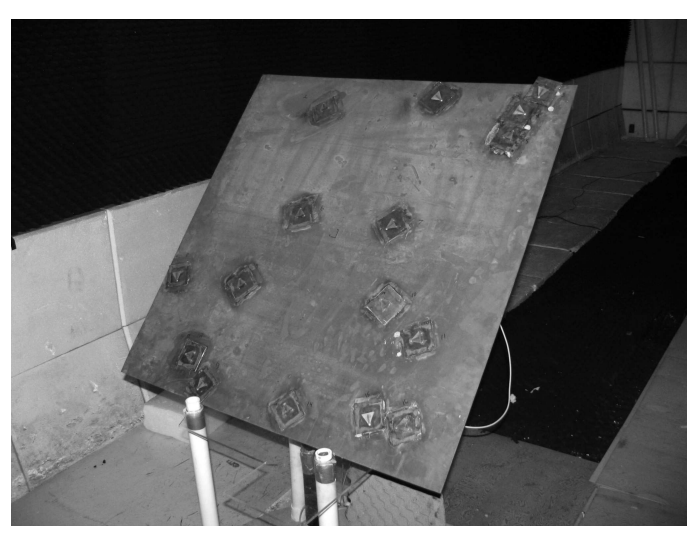

Fig. 9. Sixteen antennas mounted on ground plane which is tilted $45^{0}$ from horizontal.

where $\mu=1 / \mathbf{a}^{H} \hat{R}^{-1}$ a and $\hat{R}=1 / K \sum_{i=1}^{K} \mathbf{x}(i) \mathbf{x}(i)^{H}$. The MVDR beamformer maintains a distortionless response along the spatial signature, i.e., $\mathbf{a}^{H} \mathbf{w}=1$, while minimizing the output variance. To enhance robustness against covariance estimation error and spatial signature error, a diagonal loading term is often added to $\hat{R}$, and the loading level is conventionally chosen $10 \mathrm{~dB}$ above the noise level [6]. Once the receive beamformer is established, the transmit beamformer is obtained directly using reciprocity.

\section{Measurement Setup}

In order to emulate the scenario where sensors are randomly positioned on a surface, sixteen ESSSA's are fabricated and randomly placed on a large $1 \mathrm{~m} \mathrm{x} 1 \mathrm{~m}$ ground plane. Each antenna is also randomly rotated then soldered directly to the ground plane so that no relative shift in position can occur between antennas. A picture of the fabricated ground plane with antennas is shown in Figure 9. This array is mounted on a rotating table placed in an anechoic chamber at a 45 degree angle from horizontal. To simplify measurements, all sixteen antennas are configured for receive mode and a horn opposite the sensors is in transmit mode and remains stationary. The goal of these measurements is to transmit modulated data from the horn then measure the received I and Q baseband signals from all sensors simultaneously. An Agilent E4438C generates a $10 \mathrm{ksps}$ BPSK modulated signal using a repeating bit pattern with a $10 \mathrm{MHz}$ carrier. The E4438C signal is mixed with a $2.69 \mathrm{GHz}$ carrier then amplified and sent to the transmit horn. On the receive side an electromechanical RF switch matrix is used to select data from one antenna at a time. Every antenna sampled is subjected to an identical bit pattern so that when the data from

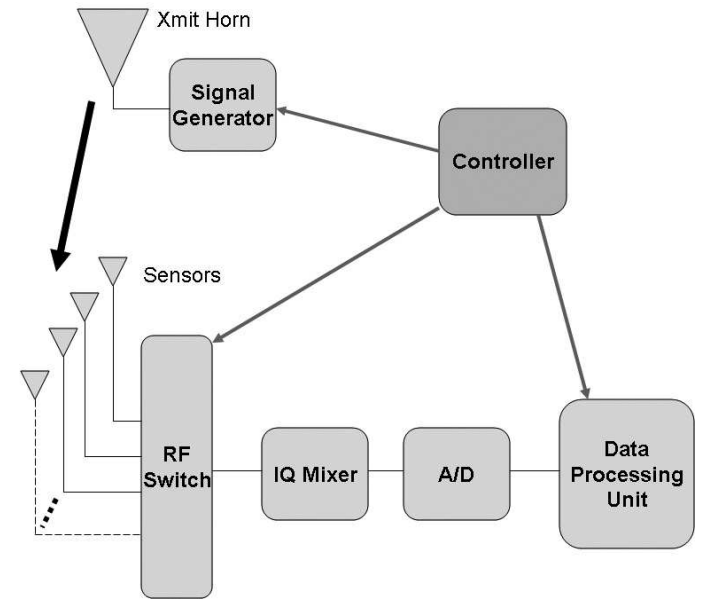

Fig. 10. Simplified measurement setup diagram

each measurement is combined it is equal to measuring all antennas simultaneously. Data from each antenna is sent via coaxial cable to the IQ mixer where it is down converted to complex baseband. The I and Q components of the complex baseband signal are sampled by the data acquisition (DAQ) board for signal processing. The DAQ board samples the I and Q signals at $50 \mathrm{kSps}$ and this data is stored on hard disk for later processing. An overview of the measurement setup is shown in Figure 10.

\section{Measured Results}

Several experiments are carried out to test the performance of the ESSSA and the beamforming algorithm. In all experiments, a bit sequence of length 1000 is encoded as an BPSK signal, modulated, and transmitted by the horn. The first 100 bits are known to the sensors for calibration purposes. The received I and Q signals are processed to recover the spatial signature and the source using the presented beamforming algorithm. The source is then decoded to reconstruct the original bit sequence. The signal-to-noise ratio (SNR) is defined as the ratio between the energy per bit and the noise level on one sensor.

\subsection{BER vS SNR}

The performance metric used in this experiment is the bit-error-rate (BER) that is defined as the percentage of erroneous bits in the recovered bit sequence. Multiple measurements are made as the power level of the transmit horn varies, which effectively varies the SNR of the sensors. For each measurement, a bit sequence is obtained and the corresponding BER is calculated. To 
vary the number of sensors used in the network, the algorithm only processes the samples received from the selected sensors, and the rest are discarded. Figure 11 shows the BER against SNR when the number of sensors in the network varies. The BER successfully decreases as more sensors are incorporated. An addition of 4 sensors to the existing network reduces BER by about $3 \mathrm{~dB}$ at $\mathrm{SNR}=-10 \mathrm{~dB}$. The improvement is more significant in a higher SNR range.

\subsection{Beampattern}

The array beampattern represents the variation in gain due to the beamformer as the angle between the sensor network and the transmitter varies. To measure the beampattern, the sensor network is first calibrated by a beacon signal to construct a beamformer that forms a beam towards the desired angle of arrival. After the beamformer is set up, the rotating table turns clockwise by an increment of $1^{0}$ at which a new set of samples are taken. The procedure is repeated until the table sweeps $180^{\circ}$ from $-90^{0}$ to $90^{\circ}$. For each degree, the samples are processed by the beamformer and the power of the beamformer output is calculated and recorded. Figure 12 shows the beampattern of an 4-element network and an 16-element network at $\mathrm{SNR}=0 \mathrm{~dB}$. The peaks of the curves are normalized to coincide at $0 \mathrm{~dB}$ for comparison purposes; the plots shows the gain due to the beamforming algorithm. Since the additional sensors provide extra degrees of freedom for noise and interference suppression, the pattern of an 16-element network has generally lower sidelobes and narrower mainlobe than that of the 4-element network. Figure 13 shows the beampatterns of the network that is calibrated to two other directions. It demonstrates that the beamforming algorithm successfully forms beams toward the direction of the beacons. While this experiment consists of only 16 sensors, actual sensor networks will have many more sensors, in which case array performance will improve.

\subsection{Time Domain Signal Waveform}

Figure 14 demonstrates three baseband signals in the time domain at SNR $=0 \mathrm{~dB}$. As shown in Figure 14(b), the received signal at one sensor is highly corrupted by noise. The beamforming algorithm combines the all the noisy inputs to form the recovered signal that sufficiently recovers the source as shown in 14(c).

\section{Conclusion}

Two important enabling technologies were presented in this paper: an electrically small antenna which min-

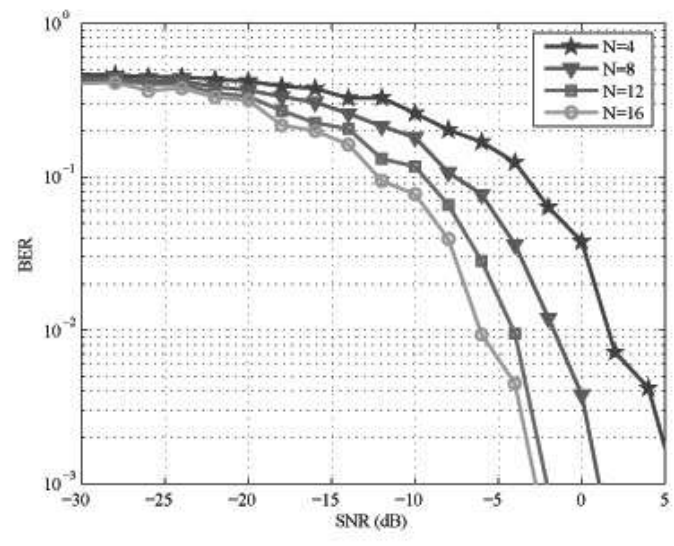

Fig. 11. Comparison of the BER performance under different number of sensors

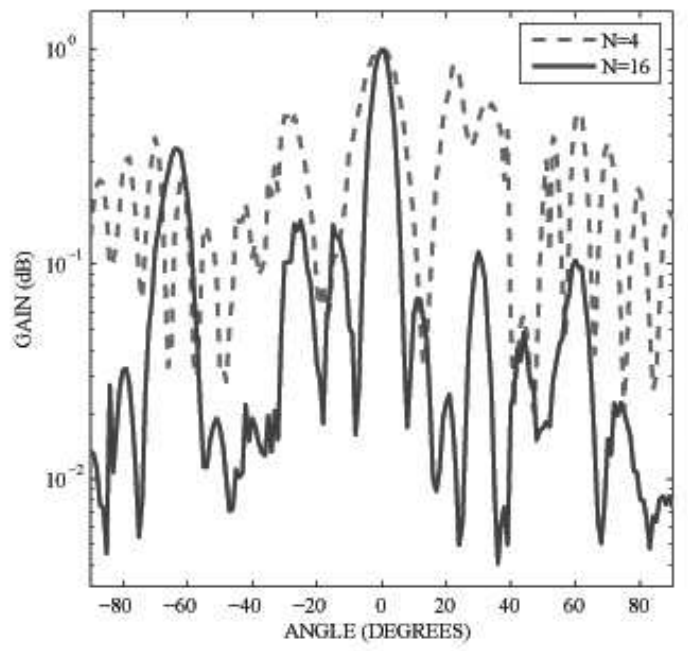

Fig. 12. Comparison of beampatterns under different number of sensors when calibrated to $0^{0}$.
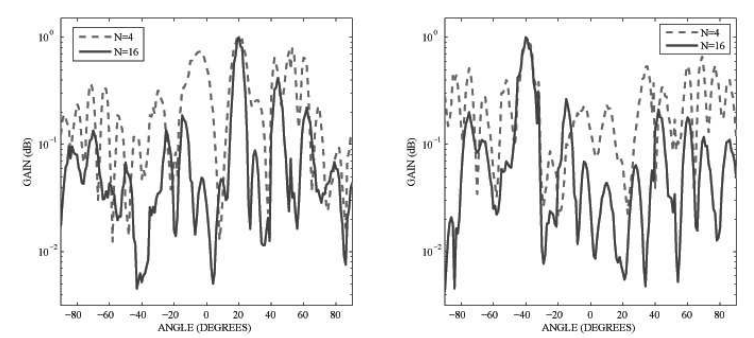

Fig. 13. Beampatterns calibrated to $20^{\circ}$ (left) and $-40^{0}$ (right). 
(a)
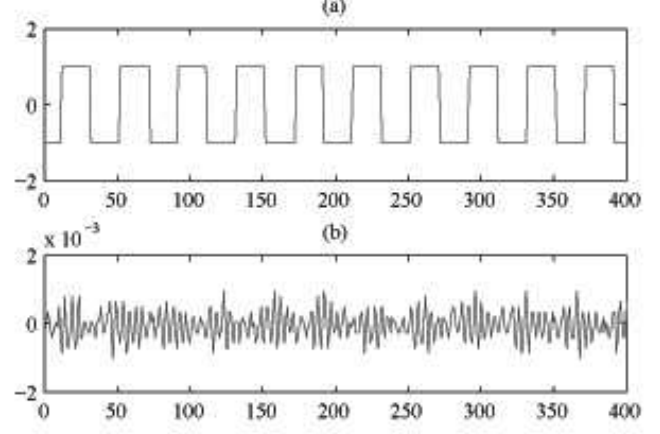

(c)

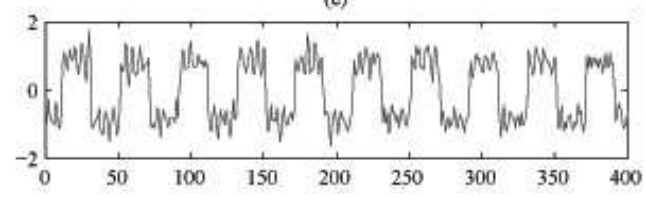

Fig. 14. (a) Source signal, (b) Real part of a complex baseband signal received from one sensor, (c) Recovered signal

imizes sensor size and beamforming algorithms which allow sensor networks to form and steer beams to orbiters or aircraft without the need for a local base station. Through use of an inductive feeding mechanism and inductive loading the stacked sector ESSSA gives $100 \mathrm{MHz}$ 2:1 VSWR impedance bandwidth while maintaining a $65 \%$ efficiency with an omni-directional radiation pattern. Measuring only $6 \mathrm{~mm}$ high, the stacked sector ESSSA is ideal for use in applications where volume is limited, especially in the vertical direction. Crucial to the operation of a sensor network, the adaptive beamforming algorithm coordinates the otherwise weak signals of individual sensors and forms one strong, steered beam in a desired direction. The results of our experiments show that even with a small array of 16 randomly positioned antennas, the beam is significantly steerable and can perform with very low bit error rates.

\section{Acknowledgments}

This work was supported by NASA through grant \#NAG3-2840.

\section{References}

[1] P. E. Mayes, "Inductively loaded, stacked sector antennas," in Proc. $25^{t} h$ Annual Applications Symposium, Allerton Park, IL, September 2001, pp. 152170.
[2] P. E. Mayes N. C. Soldner and J. T. Bernhard, "Improvement of electrically small, dual-sector antenna through integration of planar inductive feeds and inductive loads," in Proc. $29^{t} h$ Annual Applications Symposium, Allerton Park, IL, September 2005, pp. 108-126.

[3] P. E. Mayes N. C. Soldner and J. T. Bernhard, "Electrically-small inductively-loaded low-profile dual sector antennas in random arrays for surface exploration and mapping," in Antennas and Propagation Society International Symposium, July 2005, vol. 1B, pp. 516-519.

[4] H. A. Wheeler, "The radiansphere around a small antenna," Proceedings of the I.R.E., pp. 1325 - 1331, August 1959 .

[5] S. Kay, Fundamentals of Statistical Signal Processing: Estimation Theory, Englewood Cliffs, New Jersey: Prentice-Hall, 1993.

[6] H. L. Van Trees, Optimum Array Processing, New York: Wiley Interscience, 2002. 